\title{
Access to Oxazaborolidines by Catalytic Cycloaddition of 1,2-Azaborines and Vinyl Ethylene Carbonate
}

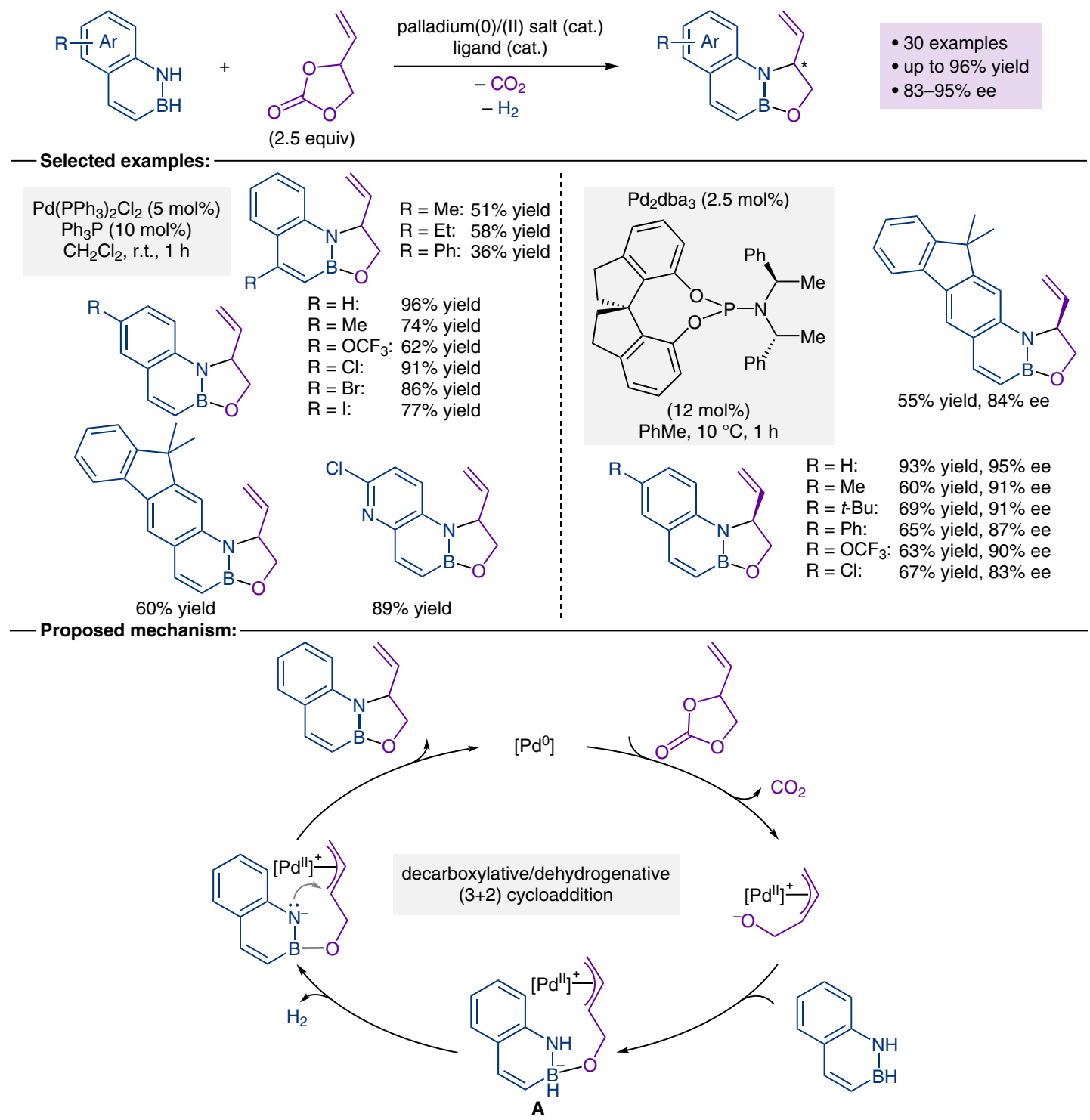

Significance: A palladium-catalyzed protocol for the (asymmetric) construction of oxazaborolidines is reported. The transformation proceeds through a decarboxylative/dehydrogenative $(3+2)$ cycloaddition of 1,2-azaborines and vinyl ethylene carbonate.
Metals in Synthesis

\section{Key words}

asymmetric synthesis

1,2-azaborines cycloaddition oxazaborolidines palladium catalysis vinylethylene carbonates

\section{Synfact is Month}

Comment: This procedure provides access to polycyclic oxazaborolidines in good yields and excellent enantioselectivities. Mechanistic experiments support the release of molecular $\mathrm{H}_{2}$ from the zwitterionic intermediate $\mathbf{A}$. 